Furthermore, qualitative evidence suggests PLFU patients felt empowered. Within palliative medicine, focus has shifted towards Phase of Illness to determine follow-up frequency, with stable patients being moved towards PLFU.

Methods A cross sectional cohort of PLFU patients is being followed for six months. Data being collected includes Phase, Karnofsky Performance Score (KPS), diagnosis, Integrated Palliative care Outcome Scale (IPOS) and outcome.

Results The project is ongoing but preliminary findings include identifying two cohorts frequently assigned to PLFU; nursing home patients, and breast cancer patients. Breast follow-up appears to be leading the way in PLFU, therefore patients transitioning from oncology follow-up to palliative care may be more amenable to a PLFU approach.

Conclusions The literature has demonstrated PLFU as a safe, cost-effective alternative to traditional follow-up and a way of ensuring a reactive service with resources to respond to crises whilst encouraging self-management and patient empowerment. Our study aims to demonstrate how PLFU translates to palliative care, and how we might identify those patients who would be candidates for this approach. It also aims to gain qualitative feedback from focus groups of both patients and relatives of deceased patients who can reflect on the transition to PLFU.

\section{EMPOWERING PATIENTS AND CARERS: A PROGRAMME TO ENCOURAGE 'THINKING AHEAD' IN THE SUPPORTIVE PHASE OF A PALLIATIVE CANCER DIAGNOSIS}

Kath Lambert, Charlotte Rock, Wendy Whitaker, Janet O'Brien. Harrogate and District NHS Foundation Trust

10.1136/bmjspcare-2019-ASP.159

Background The Harrogate and District Hospital Health and Wellbeing Project has successfully delivered education programmes to patients living with and beyond a cancer diagnosis. In 2018 the Palliative Care Team (PCT) was approached to develop a programme with focus on patients with a palliative diagnosis and their carers.

The aims of the programme include: to keep patients as well as possible for as long as possible; offer support to carers; provide information on local services; discuss advance care planning (ACP) and choices for future care.

Methods The programme is delivered by a range of professionals (e.g. PCT (Consultant and Clinical Nurse Specialist (CNS)), Psychologist, Welfare and Benefits Specialist, Carers Resource, Occupational Therapy). Referral is by Oncologist or Cancer CNS. Referral criteria includes: a palliative diagnosis for at least two months; palliative treatment or best supportive care and likely to be in the last year of life. Patients must be well enough to attend three two hour sessions over a six week period. Patients and their carer can either attend alone, together or the carer can attend alone. Maximum number of attendees is 12 per programme.

Results Three programmes have been delivered in 2018. Total number of attendees is 26 . The patients had a range of diagnoses. Drop-out for those who have attended the first session is very low. The programme has evaluated extremely well. Sessions are reported to be informative and interesting. It has facilitated discussion of ACP in an environment with attendees supporting each other. Knowledge of local services and options for future care has resulted in informed decision making.

Conclusions The 'Thinking ahead' programme will continue in 2019 with an increase in number of sessions to meet increasing referrals. The model has the potential to be developed for patients with a non-malignant diagnosis.

\section{WITHDRAWAL OF DIALYSIS; END OF LIFE CONSIDERATIONS AND ADVANCED CARE PLANNING}

J Prentice, L Hetherington, M Findlay, T Collidge. NHS Greater Glasgow and Clyde, Scottish Renal Palliative Collaberative Group, The Glasgow Renal and Transplant Unit, South Glasgow University Hospital, The Beatson West of Scotland Cancer Centre, The University of Glasgow

\subsection{6/bmjspcare-2019-ASP.160}

Background Dialysis withdrawal is the commonest cause of death in patients with end stage renal disease aged $>75$. Factors contributing the withdrawal have been described but to what degree advanced care planning (ACP) is initiated in unknown. We sought to describe current practice within a large renal unit.

Methods We performed a retrospective analysis of patients who died following dialysis withdrawal from 2015-2017. Patient demographics, markers of health, triggers for withdrawal, admissions, timing and degree of ACP discussions were extracted.

Results 52 patients were included. Median age at death 76 interquartile range (IQR) $(70,81)$ years. Median duration of dialysis was 42 (IQR 10.5, 75) months with 30.8\% dying within one year of commencement of dialysis. Median Charleston co-morbidity score was 8 (IQR 6, 9) with documentation of 'frailty' present in 52\% of the cohort. $25 \%$ of the patients had an acute event triggering withdrawal. ACP was discussed with $34 \%$ of patients with $82 \%$ of patients achieving their preferred place of death where this was documented. Median length of last admission was 24 days (IQR 11, 43) compared with 11 days (IQR 0,55) if the patient had an ACP (p-value 0.736).

Conclusion We are not good at initiating timely ACP in deteriorating dialysis patients despite identifying such patients. With an increasingly frail, multi-morbid population the need for these discussions will increase. Early ACP discussions with frail patients starting dialysis may inform later conversations and improve their quality of care. Encouragingly asking about preferred place of death improved the likelihood of achieving a death in the patients preferred location. Identification of poor prognostic markers such as dementia, introducing staff training in palliative care and adopting routine frailty scoring may guide timely conversations in anticipation of deterioration in vulnerable patient groups.

\section{THE INFLUENCE OF COMBINED ONCOLOGY AND PALLIATIVE CARE CLINICS (COPC) ON HOSPITAL ADMISSIONS}

Rachel Newman, Abbie Hewitt, Lucy Hines, Molly Jones, Shauna Green. University of Exeter Medical School, Royal Cornwall Hospitals NHS Trust, Cornwall Hospice Care

10.1136/bmjspcare-2019-ASP.161

Background Current evidence suggests early palliative care involvement (PCI) alongside oncology care improves quality of 